\title{
TECNOLOGIAS DIGITAIS E RECURSOS FÍSICOS NA ABORDAGEM DE CRIANÇAS COM TRANSTORNO DO ESPECTRO AUTISTA
}

\author{
DIGITAL AND PHYSICAL TECHNOLOGIES TO APPROACH CHILDREN WITH AUTISM SPECTRUM \\ TRANSTORN
}

\section{RECURSOS TECNOLÓGICOS DIGITALES Y FÍSICOS EN EL ENFOQUE DE NIÑOS CON TRANSTORNO DEL ESPECTRO AUTISTA}

\author{
Evellyn Ládya Franco Pontes ${ }^{1}$, Adriana Aparecida Kleparde², Taynara Pereira Silva ${ }^{3}$, \\ Ana Claudia Giesbrecht Puggina ${ }^{4}$, Maíra Rosa Apostolico5 ${ }^{5}$ Alfredo Almeida Pino-Oliveira ${ }^{6}$
}

\section{RESUMO}

Introdução: Avanços nas tecnologias digitais podem contribuir para ampliar as abordagens de educadores, profissionais de saúde e outros envolvidos no desenvolvimento integral de crianças com Transtorno do Espectro Autista (TEA). Objetivo: identificar potencialidades e aspectos comparativos entre o emprego de tecnologias digitais e de recursos físicos na abordagem de crianças com TEA. Método: Trata-se de um estudo reflexivo por meio da análise comparativa entre aplicativos exclusivamente criados para ajudar na comunicação com crianças com TEA e os recursos físicos existentes com a mesma finalidade. Resultados: Os recursos físicos apresentados são o Picture Exchange Communication System (PECS), Livro de Velcro, Troca de Figuras e Rotina Diária. Os aplicativos móveis

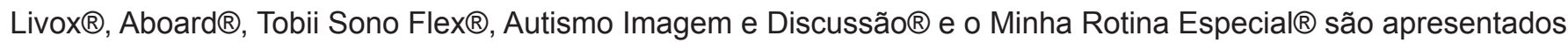
e discutidos em relação as vantagens do uso desse tipo de recurso. Conclusão: ambos os recursos podem mediar o processo de comunicação de crianças com TEA e destaca-se que os aplicativos contribuem para aprimorar a qualidade das interações realizadas com a criança com TEA.

DESCRITORES: Transtorno Autístico; Tecnologia Educacional; Tecnologia da Informação; Aplicativos Móveis.

\section{ABSTRACT}

Introduction: Advances in digital technologies may contribute to broadening the approaches of educators, health professionals and others involved in the integral development of children with Autism Spectrum Disorder (ASD). Objective: To identify potentialities and comparative aspects between the use of digital technologies and physical resources in the approach of children with ASD. Method: This is a reflective study through a comparative analysis between exclusively designed applications to help in communication with children with ASD and the existing physical resources for the same purpose. Results: The physical resources presented are the Picture Exchange Communication System (PECS), Velcro Book, Change of Figures and Daily Routine. The mobile applications Livox®, Aboard®, Tobii Sono Flex $\AA$, Autism Imaging and Discussion $\circledast$ and My Special Routine $®$ are presented and discussed concerning the advantages of using this type of resource. Conclusion: Both resources can mediate the communication process of children with $A S D$, and it is emphasized that the applications contribute to improving the quality of the interactions with the child with ASD.

DESCRIPTORS: Autistic Disorder; Educational Technology; Information Technology; Mobile Applications.

\footnotetext{
Pedagoga, Mestre em Educação. Gerente Educacional do Grupo Longevidade Saudável.

Aluna do Curso de Graduação em Pedagogia da Universidade UNIVERITAS/UNG - Guarulhos-SP

Aluna do Curso de Graduação em Pedagogia da Universidade UNIVERITAS/UNG - Guarulhos-SP

Enfermeira. Doutora em Ciências. Professora da Faculdade de Medicina de Jundiaí - FMJ

5 Enfermeira. Doutora em Ciências. Professora e Coordenadora do Programa de Pós-Graduação em Enfermagem da Universidade UNIVERITAS/UNG - Guarulhos-SP

6 Enfermeiro. Doutor em Ciências. Professor e Vice-Coordenador do Programa de Pós-Graduação em Enfermagem da Universidade UNIVERITAS/UNG - Guarulhos-SP
} 


\section{RESUMEN}

Introducción: Avances en las tecnologías digitales pueden contribuir a ampliar los enfoques de educadores, profesionales de salud y otros involucrados en el desarrollo integral de niños con trastorno del espectro autista (TEA). Objetivo: identificar potenciales y aspectos comparativos entre el empleo de tecnologías digitales y de recursos físicos en el abordaje de niños con TEA. Método: Se trata de un estudio reflexivo a través del análisis comparativo entre aplicaciones exclusivamente creadas para ayudar en la comunicación con niños con TEA y los recursos físicos existentes con el mismo propósito. Resultados: Los recursos físicos presentados son el Picture Exchange Communication System (PECS), Libro de Velcro, Intercambio de Figuras y Rutina Diaria. Las aplicaciones

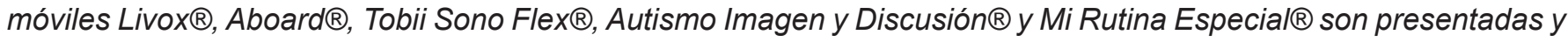
discutidas en relación con las ventajas del uso de este tipo de recurso. Conclusión: ambos recursos pueden mediar el proceso de comunicación de niños con TEA y se destaca que las aplicaciones contribuyen a mejorar la calidad de las interacciones realizadas con el niño con TEA.

DESCRIPTORES: Trastorno Autístico; Tecnología Educacional; Tecnología de la Información; Aplicaciones Móviles.

\section{INTRODUÇÃO}

Os avanços nas tecnologias digitais de informação e comunicação (TDIC) podem contribuir para ampliar as abordagens de educadores, profissionais de saúde e outros envolvidos no desenvolvimento integral de crianças com Transtorno do Espectro Autista (TEA). Tratam-se de recursos tecnológicos com potencial para promover maior interação com familiares e as próprias crianças com TEA em prol de um desenvolvimento infantil pleno, sem desconsiderar suas famílias e seus recursos comunitários para a garantia de direitos como educação, saúde, convivência social e acesso a outros serviços.

A Declaração de Salamanca ${ }^{1}$ foi redigida com a participação de 88 países incluindo o Brasil, sendo composta por documentos que asseguram a educação de pessoas com necessidades especiais e assegurando princípios, políticas e práticas alinhadas com os direitos desse grupo populacional. Aliado a esses movimentos internacionais, a promulgação da Lei Federal n 9.394, de 1996, conhecida como Lei de Diretrizes e Bases (LDB)2 , a educação inclusiva ganha maior evidência, uma vez que as instituições de ensino tornam-se responsáveis por assegurar currículos, métodos, técnicas, recursos educativos e organização específica, para melhor atender os educandos com necessidades especiais dentro de uma perspectiva comunitária que extrapola a escola e a educação formal, valorizando a inserção em outros serviços locais como saúde e desenvolvimento social, a participação das famílias e a conscientização pública.

São estes reconhecidos pela LDB como: educandos com deficiência, transtornos globais do desenvolvimento e altas habilidades ou superdotação, garantindo atendimento preferencial nas redes regulares de ensino ${ }^{2}$.
E a educação especial passa a assumir uma perspectiva de trabalhar exclusivamente com determinados alunos em escolas especializadas e com destarte, as instituições educativas têm papel central na promoção e na condução de práticas de empoderamento das crianças e das famílias para a concretização de uma sociedade inclusiva. De maneira complementar, a rede de atenção em saúde pode proporcionar espaços e parcerias para o cuidado integral e inclusivo destas crianças e suas famílias para o enfrentamento dos desafios e a construção de alternativas para lidar com os diferentes transtornos do desenvolvimento infantili,4

A quinta versão do Diagnóstico de Saúde Mental agrega o transtorno autista, transtorno de Asperger e transtorno global do desenvolvimento no Transtorno do Espectro Autista (TEA), uma vez que "os sintomas desses transtornos representam um continuum único de prejuízos com intensidades que vão de leve a grave nos domínios de comunicação social e de comportamentos restritivos e repetitivos em vez de constituir transtornos distintos" a fim de aprimorar o diagnóstico e proporcionar tratamento mais adequados ${ }^{5}$.

A educação inclusiva e especial repercute em diferentes momentos e contextos no Brasil, tendo como principal objetivo proporcionar educação para todos independente de suas especificidades ${ }^{6}$. Nesse sentido, a comunicação está entre as principais dificuldades que limitam a convivência social de crianças com TEA, pois implica na realização diversas funções no cotidiano, para solicitar algo, para negar ou expressar o que se sente, entre outras possibilidades de conhecer-se e interagir com os outros. Poucos padrões de comunicação e o grau do TEA de cada indivíduo reforçam essa limitação comunicacional. 
A Comunicação Aumentativa e Alternativa (CAA) foi traduzida do inglês "Augmentative and Alternative Communication" e pode ser identificada por meio de diferentes terminologias, quais sejam: "Comunicação Alternativa", "Comunicação Ampliada e Alternativa" ou "Comunicação Suplementar e Alternativa". Compreende um conjunto de recursos escritos, digitais, falados ou interpessoais com o objetivo de mediar a comunicação de indivíduos com transtornos de comunicação, respeitando a singularidade e a complexidade de cada ser humano no atendimento de suas necessidades específicas ${ }^{7-9}$.

Para envidar esforços nesse contexto, os profissionais da área da educação apresentam uma grande variedade de instrumentos que os auxiliam na superação de barreiras comunicacionais em prol do desenvolvimento integral de crianças com necessidades especiais. Uma das abordagens mais relevantes para a criança com TEA é o Picture Exchange Communication System (PECS) ou Sistema de Comunicação por Troca de Figuras (SCTF) $)^{8,9}$.

O PECS consiste em um recurso físico que auxilia na interação de indivíduos com dificuldades significativas na comunicação verbal ou não verbal por meio de placas com imagens e ilustrações. Algumas escolas especializadas e profissionais da área da saúde como psicólogos e fonoaudiólogos utilizam essa tecnologia para auxiliar a comunicação com esses indivíduos e facilitar a compreensão de suas necessidades e desejos.

Em suma, o setor da educação, os serviços de saúde e os espaços para o desenvolvimento social precisam acompanhar tais avanços tecnológicos, utilizando-os como facilitadores, como ferramentas proficientes e instrutivas no processo ensino-aprendizagem e no cuidado infantil integral e integrado a suas famílias e a seus recursos comunitários. Nessa perspectiva, o presente trabalho objetiva identificar potencialidades e aspectos comparativos entre o emprego de tecnologias digitais e de recursos físicos na abordagem de crianças com TEA.

\section{MÉTODO}

Trata-se de um estudo de reflexão com apoio na literatura da área de educação e tecnologias e que se baseia no levantamento intencional e não sistemático de aplicativos e recursos físicos empregados por três psicopedagogas: uma Mestre em Novas Tecnologias em Educação e com experiência docente e clínica na abordagem de crianças com TEA (ELFP) e duas Especialistas em deficiência intelectual com ênfase em autismo (AAK e TPS). Na etapa de seleção tecnológica, foram adota- dos os critérios de facilidade de acesso, gratuidade e adaptabilidade às atividades da vida diária das crianças com TEA e ou suas famílias.

Para a análise crítica e interdisciplinar das comparações entre tecnologias exclusivamente criadas para ajudar na comunicação com crianças com TEA, foram integrados à equipe de pesquisa três enfermeiros com experiência em pesquisas com interface na Educação e Saúde mediada por Tecnologias de Informação e Comunicação e na docência em Ensino Superior (AAPO, ACP e MRA) para ampliar o debate sobre as situações de aprendizagem e cuidados em saúde enriquecidas por aplicativos e PECS.

\section{RESULTADOS E DISCUSSÃO}

Para a aplicação do PECS ${ }^{3,8,9}$, o profissional capacitado neste método ensina a criança a pegar uma placa correspondente ao que ela deseja naquele momento e entregá-la ao seu parceiro de comunicação. De modo gradual e crescente, este método se amplia e pode envolver mais de uma pessoa, o que implica no ensino individualizado para a formação de frases e no respeito ao tempo e às limitações de cada criança com TEA.

Entende-se que o PECS configura uma prática incorporada em diferentes serviços que atendem crianças e familiares e apresenta seis fases que devem ser trabaIhadas de forma sequencial:

Fase I - Como se comunicar: as crianças aprendem a trocar uma única figura para itens ou atividades que eles realmente querem.

Fase II- Distância e persistência: ainda usando uma única figura, as crianças aprendem a generalizar esta nova habilidade e usá-la em lugares diferentes, com pessoas distintas e com o emprego de distâncias variadas. Elas aprendem a serem comunicadores persistentes.

Fase III - Discriminação de figuras: as crianças aprendem a escolher entre duas ou mais figuras para pedir seus itens.

Fase IV - Estrutura de sentença: as crianças aprendem a construir frases simples em uma tira de sentença usando um ícone 'Eu quero" seguido por uma figura do item que está sendo solicitado. Nessa fase, pode ocorrer o aprendizado de novos atributos e expansão de vocabulário (expansão das sentenças, acréscimo de adjetivos, verbos e preposições).

Fase V - Respondendo a perguntas: as crianças aprendem a usar o SCTF para responder à pergunta: "O que você quer?". 
Fase $\mathrm{VI}$ - Comentando: as crianças aprendem a comentar em respostas a perguntas como: "O que você vê?", "O que você ouve?" e "O que é isso?". Eles aprendem a compor sentenças começando como "Eu vejo", "Eu ouço", "Eu sinto", "É um”, entre outras.

Este sistema de figuras busca facilitar a relação interpessoal e reduzir a insatisfação do indivíduo quando não compreendido com base em símbolos simples e de fácil entendimento. Todavia, ressalta-se que a escolha das imagens deve ser adaptada de acordo com a necessidade de cada criança, sem prescindir de suas curiosidades e seus interesses. Nesse sentido, o SCTF é um recurso de baixo custo, cujos símbolos podem ser selecionados e/ou personalizados, ampliados, impressos e plastificados (Figura 1).

Figura 1 - Colagem de exemplos de Picture Exchange Communication System (PECS) ou Sistema de Comunicação por Troca de Figuras (SCTF). Guarulhos, 2018.

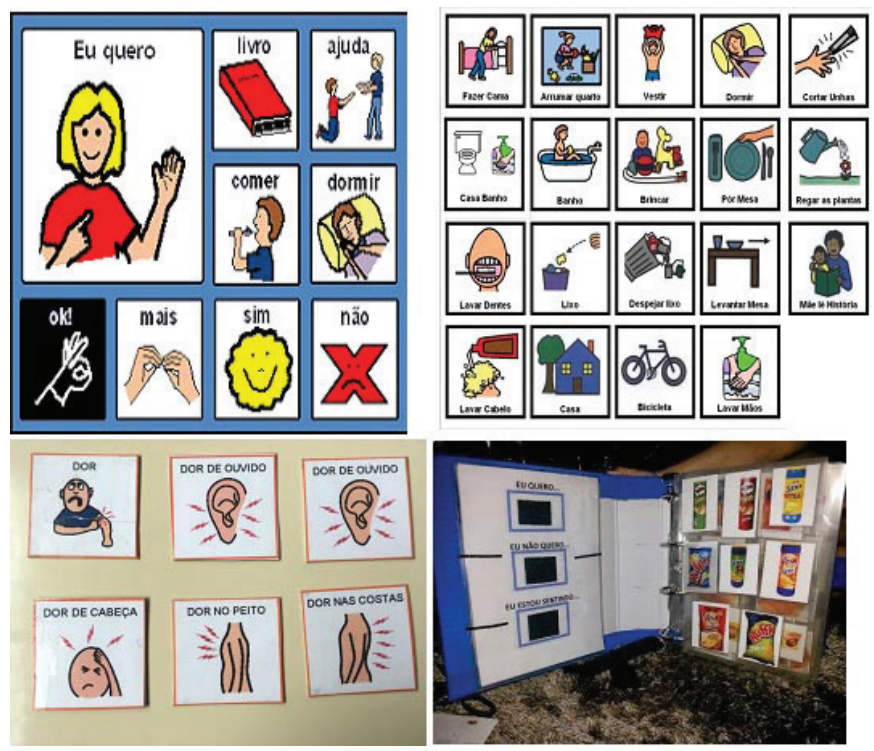

Nota: Disponível em: https://ausenda.wordpress. com/2012/11/24/pecs-sistema-de-comunicacao-por-troca-deimagens/

Cada um destes meios engajados pelo Sistema PECS apresentam funções que contribuem na comunicação e no desenvolvimento pedagógico e no cuidado integral de crianças com TEA. Para melhor compreensão sobre o uso desses recursos físicos, o quadro comparativo (Figura 2) ressalta as características e as facilidades na aplicação em abordagens pedagógicas e potencialmente interdisciplinares.
Figura 2 - Quadro comparativo entre diferentes PECS físicos. Guarulhos, 2018.

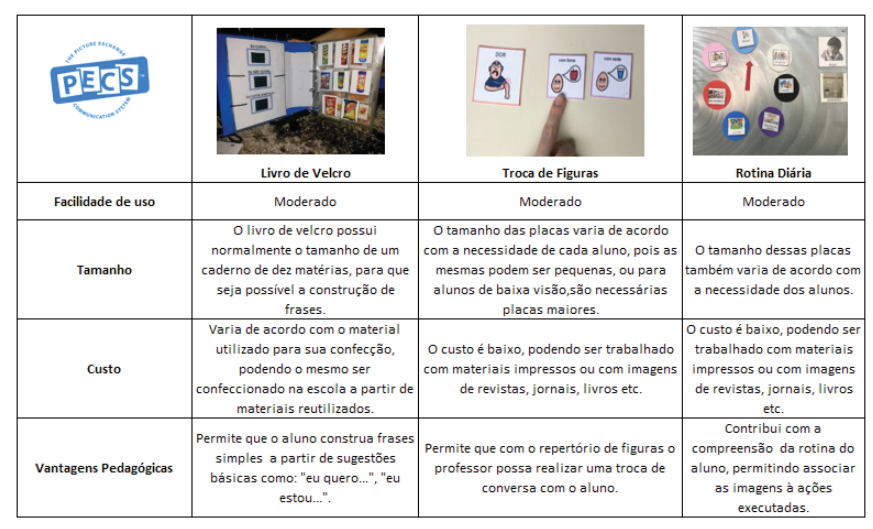

Em relação às TDIC com foco no TEA, a variedade de formas de trabalhar com as crianças pode favorecer a atuação de especialistas na CAA, mas também promove oportunidades de práticas colaborativas entre profissionais de diferentes áreas de conhecimento que necessitam abordar esse público-alvo e suas famílias ${ }^{6,8,9}$ mediadas pelo uso do Twitter $\AA^{10}$, do YouTube ${ }^{11}{ }^{11}$ e de tablets com aplicativos diversos ${ }^{12}$.

Por esse motivo, analisaram-se aplicativos, ou simplesmente Apps, em dispositivos com sistemas operacionais Android $₫$ e ou iPhone OS $₫$ (IOS). O Android® comporta três recursos específicos desse sistema operacional e que são voltados ao desenvolvimento da comunicação de indivíduos com TEA: o Livox, o Autismo Imagem e Discussão e o Aboard. Os Apps Tobii Sono Flex e o Minha Rotina Especial são compatíveis com ambos sistemas operacionais.

Elaborou-se uma síntese (Figura 3) para apresentar as funcionalidades, utilidades, aplicações e facilidades da incorporação dos aplicativos digitais na abordagem dos desejos, medos, vontades e rotinas de crianças com TEA.

Figura 3 - Quadro sintético das características dos aplicativos digitais para a abordagem de crianças com Transtorno do Espectro Autista. Guarulhos, 2018.

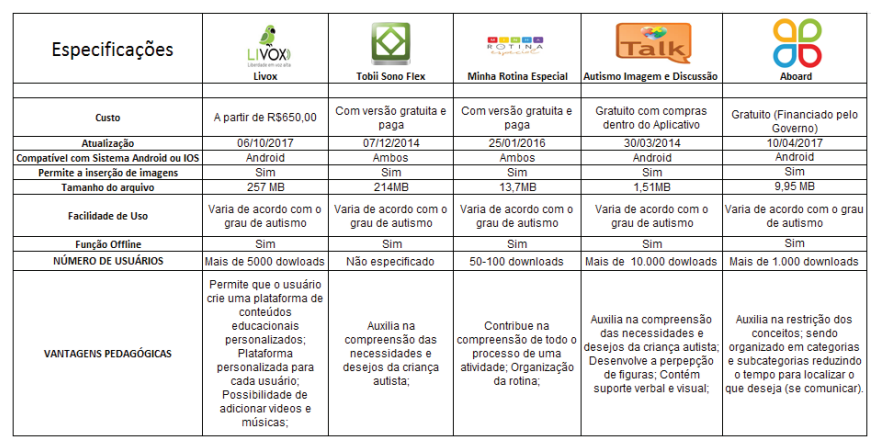

Com base nos dados acima, os aplicativos com 
maiores taxas de downloads apresentam versões gratuitas devido sua possibilidade de fácil acesso. Com relação à atualização dos Apps, poucos realizaram atualizações recentes, o que implica em uma limitação para o aperfeiçoamento constante para melhor atender as necessidades de seus usuários.

A captura e a transferência de imagens são características presentes em todos os Apps analisados e constituem um aspecto central para garantir maior personalização e contextualização na compreensão dos cotidianos de seus usuários, neste caso, as crianças com TEA supervisionadas por adultos de referência em casa, escola ou outros espaços de convívio comunitário.

Outro ponto comparado entre os Apps foi o tamanho do arquivo, uma vez que ao ocupar muito espaço de armazenamento nos celulares e outros dispositivos móveis, pode gerar uma maior dificuldade para outros downloads e desempenho dos aparelhos, em especial, quando se carregam muitas fotos como supracitado.

Sobre a questão das vantagens pedagógicas, o Livox® permite que o usuário crie uma plataforma de conteúdos educacionais personalizada com articulação, fluência e clareza na linguagem, além de promover a comunicação por meio de frases usando palavras rápidas e de diferentes categorias semânticas.

O Aboard $®$ e o Tobii Sono Flex® também favorecem a construção de frases. O Autismo Imagem e Discussão ${ }^{\circ}$ auxilia o professor na compreensão das necessidades e desejos do aluno por meio da aplicação de figuras. O Minha Rotina Especial $®$ permite a organização das atividades cotidianas baseada em imagens focadas nos interesses do próprio usuário.

Defende-se aqui que a utilização de ferramentas digitais como facilitador na comunicação de crianças com TEA para ampliar e fortalecer a CAA entre diferentes profissionais, as próprias crianças e seus familiares. Compará-las com o PECS (Figura 4) pode ajudar na seleção mais adequada do recurso tecnológico (impresso, analógico e ou digital) na promoção da aprendizagem, do cuidado em saúde, do desenvolvimento humano integral e no exercício da cidadania.
Figura 4 - Quadro comparativo entre PECS e aplicativos digitais. Guarulhos, 2018.

\begin{tabular}{|c|c|c|c|c|c|}
\hline Especificações & Livox & $\underset{\text { Totii Sono flex }}{0}$ & 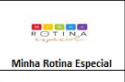 & $\underset{\text { Autismo Imagem e Discussão }}{\text { Talk }}$ & $\mathrm{O}_{\text {Aboard }}^{90}$ \\
\hline custo & Apartit de R\$650000 & $\begin{array}{c}\text { Comversão oratuita } \\
\text { paga }\end{array}$ & $\begin{array}{c}\text { Com versão oratuta o } \\
\text { paga }\end{array}$ & $\begin{array}{l}\text { Gratuito com comprass } \\
\text { dentro do Aplicativo }\end{array}$ & $\begin{array}{c}\begin{array}{c}\text { Gratuito (Financlado } \\
\text { Governo) }\end{array} \\
\end{array}$ \\
\hline $\begin{array}{l}\text { Atualizaçäo } \\
\text { Com Sistema An }\end{array}$ & $\begin{array}{l}\frac{061102017}{2} \\
\text { Android } \\
\end{array}$ & $\begin{array}{c}07 / 12212014 \\
\text { Ambos } \\
\end{array}$ & $\begin{array}{c}2510122016 \\
\text { Ambos } \\
\end{array}$ & $\begin{array}{l}\frac{30 / 10322014}{\text { Android }} \\
\end{array}$ & $\begin{array}{l}\text { 10:04:42017 } \\
\text { Android }\end{array}$ \\
\hline $\begin{array}{l}\text { Permite a inserer,jö de imagens } \\
\text { Tamanho do arcuivo }\end{array}$ & $\begin{array}{l}\text { Sim } \\
257 \mathrm{MB}\end{array}$ & $\begin{array}{l}1 m \\
214 \mathrm{MB} \\
\end{array}$ & $\begin{array}{l}\mathrm{sim} \\
13.7 \mathrm{MB} \\
\end{array}$ & $\begin{array}{l}\frac{51 \mathrm{~m}}{1.51 \mathrm{MB}} \\
\end{array}$ & $\begin{array}{l}\text { Sim } \\
.95 \mathrm{ME} \\
\end{array}$ \\
\hline Fachiliasate de SSo & $\begin{array}{l}\text { Varia de acordo como } \\
\text { grau de autismo }\end{array}$ & $\begin{array}{l}\text { Vaira de acordo como } \\
\text { grau de auismo } \\
\text { ans }\end{array}$ & $\begin{array}{l}\text { Varia de acordo comom } \\
\text { grau de autismo }\end{array}$ & $\begin{array}{l}\text { Yairia de acordo como } \\
\text { grau de autismo }\end{array}$ & $\begin{array}{l}\text { Varia de acordo com o crau } \\
\text { de autismo }\end{array}$ \\
\hline & sim & sim & & $\operatorname{sim}$ & sim \\
\hline VANTIAGENS PEDAGOGGICAS & 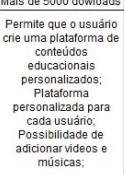 & $\begin{array}{l}\text { Auxilia na } \\
\text { compreensád das } \\
\text { necessidades e } \\
\text { desejos da crianca } \\
\text { autista; }\end{array}$ & 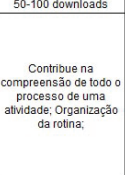 & 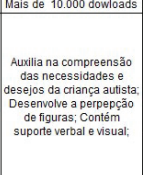 & 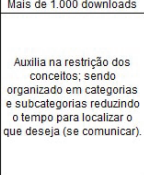 \\
\hline
\end{tabular}

Ambas as categorias permitem a atualização e ou adaptação das figuras. No entanto, o PECS exige um procedimento mais detalhado incluindo: a captura da imagem, a impressão (ou recorte), a plastificação (visando maior durabilidade das plaquinhas) e a colagem do velcro (no caso de trabalhar com o livro de velcro).

Esses processos tornam sua produção trabalhosa e podem reduzir a autonomia da criança com TEA. Contudo, ressalta-se que valorizar a participação ativa infantil na elaboração de seus recursos pedagógicos não exclui a importância de um maior acompanhamento dos adultos de referência em casa, na escola, nas unidades de saúde e outros centros de referência.

No caso dos Apps, a presença do adulto de referência é essencial. Instalar, manusear, interagir, pesquisar imagens, pagar por determinadas funções (se necessário ou desejado) exemplificam ações que indicam a necessidade de acompanhamento adulto para que as ferramentas digitais sejam bem utilizadas e incorporadas pelas crianças com TEA.

Em relação à facilidade na compreensão das imagens, os Apps utilizam um sistema visual e verbal de imagens (disponíveis na internet, compartilhadas ou autorais) que atribui maior significado para o desenvolvimento da percepção e da compreensão da criança com TEA, bem como tornar o processo de comunicação mais seguro e assertivo. Por outro lado, algumas placas no PECS são ilustradas previamente e, muitas vezes, podem dificultar a aproximação de elementos da realidade concreta da própria criança.

Para o manuseio e transporte dos recursos, o PECS apresenta menor disponibilidade no tempo, conteúdo das imagens e lugar quando comparado aos Apps. Entende-se que para o bom desenvolvimento da comunicação com a criança com TEA, as placas precisam estar à disposição dela. Caso a criança com TEA se encontre em um ambiente diferente do seu cotidiano ou 
não identifique imagens específicas, a frustração é muito frequente. Acrescenta-se que as tecnologias digitais armazenadas em diferentes dispositivos móveis superam tais dificuldades, mas exigem a assistência de um adulto letrado digitalmente, especialmente nos casos mais graves de TEA, para aplicá-las adequadamente.

Quando se consideram os requisitos de ajustes, os Apps dependem da velocidade de atualização em seus sistemas operacionais, enquanto o PECS permite essa construção em "tempo real" de novas placas de figuras com base na ampliação do repertório na comunicação da criança com TEA. Esse processo pode ser mais rápido ou lento, a depender do tempo que o profissional terá para pesquisa, produzir, recortar ou imprimir esse recurso tecnológico.

No tocante a questões financeiras, ambos os recursos apresentam baixo custo, pois os valores do PECS variam de acordo com os materiais selecionados, podendo ser feitos com materiais reutilizáveis e com o conhecimento e força de trabalho de seus próprios utilizadores. A gratuidade no uso dos Apps favorece sua obtenção e potencial aplicação. Quatro dos cinco aplicativos analisados são totalmente gratuitos, um deles apresenta uma versão gratuita e algumas funcionalidades pagas.

Reforça-se que a CAA objetiva proporcionar ao indivíduo maior autonomia na comunicação verbal ou não-verbal7 ${ }^{7,9,13}$. Nesse sentido, constata-se que os recursos digitais se sobrepõem aos recursos físicos, uma vez que a onipresença e a expansão das TDIC no cotidiano da criança, da família e da sociedade implicam na diversificação da função visual, sonora e escrita. Os Apps possibilitam que figuras e palavras sejam enunciadas, favorecendo que a criança com TEA desenvolva o reconhecimento de cada objeto e possa pronunciar algumas sílabas ou até mesmo palavras.

Para os professores, os Apps podem tornar suas aulas mais interativas em virtude da motivação favorecida pelo elemento digital na curiosidade da criança com TEA e, sempre que possível, criar oportunidades para maior engajamento da família e de outros representantes significativos de sua comunidade. Analogamente, profissionais de saúde, da assistência social e outras áreas podem ponderar as vantagens e desvantagens do uso dessas TDIC na promoção do cuidado integral e do desenvolvimento humano pleno.

Em contrapartida ao PECS, as funcionalidades inclusivas e potencialmente significativas das TDIC para o processo de aprendizagem e do cuidado integral das crianças com TEA. Estas ferramentas digitais valorizam formas lúdicas, instigam a curiosidade e proporcionam novas oportunidades para ensinar, aprender, cuidar e interagir em uma sociedade predominantemente digital em seus processos de comunicação ${ }^{12,14-16}$.

\section{CONSIDERAÇÕES FINAIS}

O presente trabalho evidencia que as TDIC podem contribuir sobremaneira para a ampliação do repertório de diferentes profissionais de educação, saúde e assistência social para promover a comunicação e a interação com crianças com TEA e seus familiares, sem prescindir de seus recursos comunitários (presenciais e virtuais).

A seleção dos recursos físicos (PECS) e recursos digitais (Apps) a fim de compreender suas facilidades e principais características permitiu uma análise comparativa para ajudar na avaliação crítica e contextualizada dos recursos tecnológicos mais relevantes para sua incorporação personalizada na rotina da criança com TEA pelos professores, psicólogos, fonoaudiólogos, enfermeiros, assistentes sociais, entre outros atores sociais estratégicos.

Conclui-se que ambos os recursos podem mediar o processo de comunicação de crianças com TEA. Destaca-se que os Apps contribuem para aprimorar a qualidade das interações realizadas com a criança com TEA a fim de proporcionar novos caminhos para o desenvolvimento da aprendizagem e do cuidado integral que utilizam diferentes canais de comunicação visual, sonora e escrita para a atuação profissional e ou apoio às famílias.

À guisa de síntese provisória, espera-se que essa atualização subsidie práticas interdisciplinares enriquecidas por TDIC e que enfatizam o fortalecimento da autonomia da criança com TEA, do apoio a seus familiares e do melhor desenvolvimento humano integral possível em uma sociedade cada vez mais digital e potencialmente inclusiva. 


\section{Referências}

1. ONU. Declaração de Salamanca: sobre princípios, políticas e práticas na área das necessidades educativas especiais [Internet]. Salamanca; 1994 [cited 2018 Dec 31]. Available from: http://portal.mec.gov. $\mathrm{br} /$ seesp/arquivos/pdf/salamanca.pdf

2. Brasil. Lei № 9.394 de 20 de dezembro de 1996 que estabelece as diretrizes e bases da educação nacional. [Internet]. Diário Oficial da União, 93941996 [cited 2018 Dec 31] p. 1-28. Available from: http://portal.mec.gov.br/seesp/arquivos/pdf/ lei9394_Idbn1.pdf

3. Neves AJ das, Antonelli C de S, Silva MGC da, Capellini VLMF. Escolarização formal e dimensões curriculares para alunos com autismo: o estado da arte da produção acadêmica brasileira. Educ em Rev [Internet]. 2014 [cited 2018 Dec 31];30(2):4370. Available from: http://www.scielo.br/pdf/edur/ v30n2/03.pdf

4. Gomes PTM, Lima LHL, Bueno MKG, Araújo LA, Souza NM, Gomes PTM, et al. Autism in Brazil: a systematic review of family challenges and coping strategies. J Pediatr (Rio J) [Internet]. Sociedade Brasileira de Pediatria; 2015 Mar [cited 2018 Dec 31];91(2):111-21. Available from: https://linkinghub. elsevier.com/retrieve/pii/S002175571400165X

5. American Psychiatric Association. Manual diagnóstico e estatístico de transtornos mentais: DSM-5 [Internet]. 5th ed. Porto Alegre; 2014 [cited 2018 Dec 31]. 992 p. Available from: https://aempreendedora.com.br/wp-content/uploads/2017/04/ Manual-Diagnóstico-e-Estatístico-de-Transtornos-Mentais-DSM-5.pdf

6. Santarosa LMC, Conforto D. Tecnologias móveis na inclusão escolar e digital de estudantes com transtornos de espectro autista. Rev Bras Educ Espec. 2015; 21(4):349-66.

7. Lund SK, Quach W, Weissling K, McKelvey M, Dietz $A$, et al. Assessment with children who need augmentative and alternative communication (AAC): Clinical decisions of AAC specialists. Lang Speech Hear Serv Sch. 2017; 48(1):56-68.

8. Oliveira TP, Jesus JC. Analysis of an alternative communicative training for teaching request to autistic. Psic da Educ [Internet]. 2016 [cited 2018 May 19];42:1-23. Available from: http://pepsic.bvsalud. org/pdf/psie/n42/n42a03.pdf

9. Ferreira $C$, Bevilacqua $M$, Ishihara M, Fiori $A, A r-$ monia A, Perissinoto J, et al. Seleção de vocábulos para implementação do Picture Exchange Communication System - PECS em autistas não verbais Selection of words for implementation of the Picture Exchange Communication System - PECS in non-verbal autistic children. CoDAS [Internet]. 2017 [cited 2018 May 19];29(1). Available from: http://www.scielo.br/pdf/codas/v29n1/2317-1782-codas-2317-178220172015285.pdf

10. Hemsley B, Palmer S, Dann S, Balandin S. Using Twitter to access the human right of communication for people who use Augmentative and Alternative Communication (AAC). Int J Speech Lang Pathol; 2018.

11. Azer SA, Bokhari RA, Alsaleh GS, Alabdulaaly MM, Ateeq KI, Guerrero APS, et al. Experience of parents of children with autism on YouTube: are there educationally useful videos? Informatics Heal Soc Care [Internet]. 2018 [cited 2018 Dec 31];1-15. Available from: http://www.tandfonline.com/action/ journallnformation?journalCode=imif20

12. Lorah ER, Parnell A, Whitby PS, Hantula D. A systematic review of tablet computers and portable media players as speech generating devices for individuals with Autism Spectrum Disorder. J Autism Dev Disord. 2015; 45:3792-804.

13. Assar S. Information and communications technology in Education. Int Encycl Soc Behav Sci [Internet]. Elsevier; 2015 Jan 1 [cited 2018 Dec 17];6671. Available from: https://www.sciencedirect.com/ science/article/pii/B9780080970868921044

14. Agarwal $S$, Lefevre $A E$, Labrique $A B$. A call to digital health practitioners: new guidelines can help improve the quality of digital health evidence. JMIR mHealth uHealth [Internet]. JMIR Publications Inc.; 2017 Oct 6 [cited 2018 May 20];5(10):e136. Available from: http://www.ncbi.nlm.nih.gov/pubmed/28986340

15. Rossi LP, Lovisi GM, Abelha L, Gomide M. Caminhos virtuais e autismo: acesso aos serviços de saúde na perspectiva da análise de redes sociais. Cien Saude Colet [Internet]. ABRASCO - Associação Brasileira de Saúde Coletiva; 2018 Oct [cited 2018 Dec 31];23(10):3319-26. Available from: http://www.scielo.br/scielo.php?script=sci_ arttext\&pid=S1413-81232018001003319\&lng=pt\&t Ing=pt

16. Pontes ÉLF. Cultura digital na formação inicial de pedagogos. São Paulo: Artesanato Educacional; 2018. 204 p. 